\title{
Pulmoner emboli tanısında D-dimer düzeyleri ile ventilasyon/perfüzyon sintigrafisindeki bulgular arasındaki ilişki
}

\author{
Relationship between scintigraphic findings in ventilation/perfusion \\ scintigraphy and D-dimer levels in diagnosis of pulmonary embolism \\ Zekiye Hasbek*, Taner Erselcan, Serdar Savaş Gül \\ Nükleer Tıp Anabilim Dalı (Yrd. Doç Dr. Z. Hasbek, Prof. Dr. T. Erselcan), Cumhuriyet \\ Üniversitesi Tıp Fakültesi, TR-58140 Sivas, Nükleer Tıp Anabilim Dalı (Yrd. Doç. S. S. Gül), \\ Gaziosmanpaşa Üniversitesi Tıp Fakültesi, TR-60100 Tokat
}

\begin{abstract}
Özet
Amaç. Pulmoner emboli (PE) ölüm riskinin yüksek olması ve PE kuşkusu ile başlanan antikoagülan tedavinin yüksek kanama riski taşıması, tanının en kısa sürede doğrulanmasını ya da dışlanmasını gerektiren kardiovasküler hastalıktır. Ventilasyon/perfüzyon (V/P) sintigrafisi PE şüphesinde yaygın kullanılan testlerden biridir. D-dimer fibrin yıkımının bir ürünüdür. Ancak akut trombozlar için spesifik değildir. Bu çalışmada amacımız, D-dimer düzeyleri ile ventilasyon/perfüzyon sintigrafisindeki perfüzyon defekt sayısı arasındaki ilişkiyi araştırmaktı. Yöntem. Çalışmamızda, PE ön tanısı ile takip edilen 100 olgunun D-dimer düzeyleri ile V/P sintigrafileri arasındaki ilişki araştırıldı. Hastaların sintigrafik bulguları perfüzyon defekti açısından; "perfüzyon defekti yok", "subsegmenter tutulum", "bir segment tutulum", "iki segment tutulum", "üç segment tutulum" ve "üçten fazla segment tutulum" şeklinde altı gruba; pulmoner emboli olasılığı açısından ise "pulmoner emboli yok", "düşük olasılıklı", "orta olasıllklı" ve "yüksek olasılıklı" şeklinde dört gruba ayrıldı. D-dimer düzeyi referans aralığı 0,00-0,50 $\mu \mathrm{g} / \mathrm{mL}$ idi. Bulgular. Çalışmaya alınan 100 hastanın 52'si erkek 48'i kadındı. Pulmoner emboli olmayan 65 hastada D-dimer: 4,39 $\pm 9 \mu \mathrm{g} / \mathrm{mL}$; düşük olasılıklı 20 hastada D-dimer: 4,57 $\pm 4 \mu \mathrm{g} / \mathrm{mL}$; orta olasılıklı olan 9 hastada D-dimer: 6,38 $\pm 6 \mu \mathrm{g} / \mathrm{mL}$; yüksek olasilıklı olan 6 hastada D-dimer: $8.0 \pm$ $8 \mu \mathrm{g} / \mathrm{mL}$ olarak bulundu ( $>0,05)$. Perfüzyon defekti izlenen segment sayısına göre; perfüzyon defekti olmayan 22 hastada D-dimer: 3,01 $\pm 4 \mu \mathrm{g} / \mathrm{mL}$; subsegmenter defekti olan 8 hastada Ddimer: 3,62 $\pm 3 \mu \mathrm{g} / \mathrm{mL} ; 1$ segment defekti olan 14 hastada D-dimer: $10,7 \pm 18 \mu \mathrm{g} / \mathrm{mL} ; 2$ segment defekti olan 11 hastada D-dimer: 3,16 $\pm 2 \mu \mathrm{g} / \mathrm{mL} ; 3$ segment defekti olan 10 hastada D-dimer: $3,86 \pm 3 \mu \mathrm{g} / \mathrm{mL}$; 3 'ten fazla segment defekti olan 35 hastada D-dimer: 4,66 $\pm 4 \mu \mathrm{g} / \mathrm{mL}$ olarak bulundu $(\mathrm{p}<0,05)$. Sonuç. D-dimer düzeyi ile ventilasyon/perfüzyon sintigrafisindeki sintigrafik bulguların ilişkisini incelediğimiz çalışmamızın sonucunda, D-dimer seviyesi bir segmentte perfüzyon defekti olan grupta yüksek, buna karşın üçten fazla segmentte tutulum olan grupta ise daha düşük olarak bulundu. Bu durum, tek segment defekti olan vakaların gerçek akut PE ile uyumlu vakalar olması ve daha fazla sayıda segment defekti olanların ise daha çok kronik hastalıkları bulunan vakalar olduğunu düşündürmektedir.
\end{abstract}

Anahtar sözcükler: D-Dimer, pulmoner emboli, ventilasyon/perfüzyon sintigrafi

\begin{abstract}
Aim. Pulmonary embolism (PE) is a cardiovascular disease that the diagnosis is needed urgent recognition or exclusion, since the PE has high risk of death and anticoagulant treatment starting with suspicion of PE has high risk of bleeding. Ventilation/perfusion(V/P) scintigraphy is one of the widely used tests in suspect of PE. D-dimer is a product of fibrin degradation. However, it is not specific for acute thrombosis. The purpose of this study was to investigate the relation between number of the defect in the ventilation/perfusion scintigraphy and D-dimer levels. Method. In our study, the relation between D-dimer levels of 100 cases followed-up with PE pre-diagnosis and their V/P scintigraphy was investigated. Scintigraphic findings of the patients were divided into six groups regarding the perfusion detect as; "no involvement", "subsegmenter-involvement", "onesegment involvement", "two-segments involvement", "three-segments involvement", "more than three-segments involvement" and also divided into four groups regarding the possibility of
\end{abstract}


pulmonary as; "no pulmonary embolism", "low-probability", "intermediate-probability" and "high-probability". The reference range of D-dimer level was $0.00-0.50 \mu \mathrm{g} / \mathrm{mL}$. Results. Of the 100 patients studied on, 52 were male and 48 were female. In 65 patients without pulmonary embolism D-dimer: $4.39 \pm 9 \mu \mathrm{g} / \mathrm{mL}$; in 20 patients with low-probability D-dimer: $4.57 \pm 4$ $\mu \mathrm{g} / \mathrm{mL}$;in 9 patients with intermediate-probability D-dimer: $6.38 \pm 6 \mu \mathrm{g} / \mathrm{mL}$ and, in 6 patients with high-probability D-dimer:8.0 $\pm 8 \mu \mathrm{g} / \mathrm{mL}(\mathrm{p}>0.05)$. Regarding the number of segment monitored perfusion defect; in 22 patients without perfusion defect D-dimer: $3.01 \pm 4 \mu \mathrm{g} / \mathrm{mL}$;in 8 patients with subsegmental defect D-dimer: $3.62 \pm 3 \mu \mathrm{g} / \mathrm{mL}$; in 14 patients with one segment defect Ddimer: $10.7 \pm 18 \mu \mathrm{g} / \mathrm{mL}$; in 11 patients with 2 segments defect D-dimer: $3.16 \pm 2 \mu \mathrm{g} / \mathrm{mL}$; in 10 patients with 3 segments defect D-dimer: $3.86 \pm 3 \mu \mathrm{g} / \mathrm{mL}$, in 35 patients with more than 3 segments defect D-dimer: $4.66 \pm 4 \mu \mathrm{g} / \mathrm{mL}(\mathrm{p}<0.05)$. Conclusion. In consequence of our study that we researched relations between D-dimer levels and scintigraphic findings in ventilation/perfusion scintigraphy; D-dimer level was found high in the group with perfusion defect in one segment, but however it was found lower at the group in more than three segments defects. This situation makes us think that the cases with one segment defect are ones coherent with real acute PE, and the cases with more segment defects are the cases who rather has chronic diseases.

Keywords: D-Dimer, pulmonary embolism, ventilation/perfusion scintigraphy

Geliş tarihi/Received: 03 Haziran 2014; Kabul tarihi/Accepted: 23 Haziran 2014

\section{*Iletișim adresi:}

Dr. Zekiye Hasbek, Nükleer Tıp Anabilim Dalı, Cumhuriyet Üniversitesi Tıp Fakültesi, TR-58140 Sivas. E-posta: hasbekz@yahoo.com

\section{Giriş}

Pulmoner emboli, pulmoner arter veya dallarının hematojen yol ile gelen trombüs, hava, yağ, parazit ve tümöral doku parçaları ile aniden tıkanması ile oluşan ve son 30 yılda teşhis ve tedavideki ilerlemelere rağmen, hala yüksek mortalite hızı olan akut kardiyovasküler hastalıktır. Yetişkin yaştaki ölümlerin \%10-15'inden sorumludur [1]. Tedavi edilmemiş olgularda PE'nin mortalitesi yaklaşık \%25-30 iken, tedavi edilenlerde mortalite \%2-8'e düşer [2]. Mortalite genellikle ileri yaş, kardiovasküler komorbidite ve kanserli hastalarda sıktır [3]. Pulmoner emboli varlığında, pulmoner yataktaki tıkanıklık nedeniyle akut sağ ventrikül yetmezliği gelişebiliceğinden erken teşhis oldukça önemlidir. Çünkü tedavi edilmeyen hastaların çoğu tanıdan sonraki ilk birkaç saat içerisinde kaybedilir. Oysa tedavi yüksek oranda efektiftir ve sağ ventrikül yetmezliği geri dönüşlüdür. Vakaların \%85'inden fazlasında pulmoner embolinin kaynağı özellikle inferior vena kava, iliak ve renal venlerden kaynaklanan derin ven trombozudur [4]. Kadın olgularda en önemli kaynak pelvik venlerdir. Bazen aksiler, subklavian venler, sağ kalp kapak ve boşlukları da emboli kaynağı olabilir [2].

Pulmoner embolide klinik ve fizik muayene bulguları özgül değildir. Tıkanan damarın çapı ne kadar geniş olursa, pulmoner embolinin kliniği de o kadar ağır olur. Klasik semptomlar; dispne, takipne, taşikardi ve gögüs ağrısıdır. Öksürük, hemoptizi ve senkop diğer görülen semptomlardır. Çok ciddi vakalar kardiak arrest, şok veya hipotansiyonla gelebilir.

Klinik olarak şüphelenilen hastaların tanısında EKG, direk grafi, laboratuvar testleri (Ddimer ve kardiak belirteçler), görüntüleme teknikleri (en sık olarak BT pulmoner anjiografi ve ventilasyon-perfüzyon sintigrafisi) ve ekokardiografi yapılır. Endojen fibrinolitik sistemin yeni oluşmuş trombüsü parçalaması sonucu salınan bir fibrin yıkım ürünü olan D-dimer, PE kuşkusu durumunda tanıya yardımcı olarak en sık kullanılan laboratuvar tanı testlerindendir.

Bizim bu çalışmadaki amacımız; D-dimer düzeyleri ile ventilasyon/perfüzyon sintigrafisindeki perfüzyon defekt sayısı arasındaki ilişkiyi araştırmaktı. Bu nedenle makalemizde yalnızca bu iki grup tanı testini tartıştık. 


\section{Gereç ve yöntem}

$\mathrm{Bu}$ çalışmaya, PE ön tanısı ile izlenen, bölümümüze V/P sintigrafi istemi ile gönderilen ve tanı için D-dimer düzeyleri çalışılmış olan 100 hasta dahil edildi. Hematolojik, hepatik ve renal hastalığ zamanda cerrahi operasyon geçiren hastalar çalışmaya dahil edilmedi. Retrospektif olan bu çalışma, Helsinki Deklarasyonu etik standartlarına uygun olarak yapıldı.

Perfüzyon sintigrafisi, hasta yatar pozisyonda iken Tc-99m LyoMAA (Human albumin macroagregate-Mallinckrodt, Hazelwood, MO, USA) ortalama 74-185 MBq dozunda yavaş olarak i.v. olarak enjekte edilmesinin ardından çekildi. Çift başlı (ECAM-Toshiba) gama kamerada düşük enerjili genel amaçlı kolimatör (LEAP) kullanılarak, 128 x 128 matrikste, anterior, posterior, sağ lateral, sol lateral, sağ posterior oblik, sol posterior oblik, sağ anterior oblik ve sol anterior oblik pozisyonlarda olmak üzere statik görüntüler elde edildi. Bir gün sonra yapılan ventilasyon sintigrafisi ise Tc-99m DTPA'nın (dietilentriaminpentaasetik asit) ortalama 555-925 MBq dozunda nebulizatör yardımıyla yaklaşık 15 dakika süre ile inhale ettirilmesinin ardından çekildi. Çift başlı (ECAMToshiba) gama kamerada düşük enerjili genel amaçlı kollimatör (LEAP) kullanılarak, $128 \times 128$ matrikste, anterior, posterior, sağ lateral, sol lateral, sağ posterior oblik, sol posterior oblik, sağ anterior oblik ve sol anterior oblik olmak üzere perfüzyon sintigrafisi ile aynı pozisyonlarda statik görüntüler elde edildi.

Hastalar perfüzyon sintigrafisinde tespit edilen perfüzyon defekti açısından "perfüzyon defekti yok", "subsegmenter tutulum", "bir segment tutulum", "iki segment tutulum", "üç segment tutulum" ve "üçten fazla tutulum" şeklinde altı gruba ayrıldı. Ayrıca pulmoner emboli açısından PİOPED (prospective investigation of pulmonary embolism diagnosis) kriterlerine göre ise "pulmoner emboli yok", "düşük olasılıklı", "orta olasılıklı" ve "yüksek olasılıklı" şeklinde dört gruba ayrıldı [5]. Bunun dışında hastalar 20-44 yaş, 4564 yaş ve 65 yaş ve üzeri olmak üzere üç gruba ayrildı.

D-dimer düzeyi STA compact (diagnostica stago ${ }^{\circledR}$ ) cihazı ile Liatest ${ }^{\circledR}$ DD-I kiti kullanılarak tayin edildi (Referans aralığı: 0,00-0,50 $\mu \mathrm{g} / \mathrm{mL}$ ).

Verilerin istatistiksel analizi, SPSS 14,0 kullanılarak yapıldı ve $\mathrm{p}<0,05$ anlamlı olarak kabul edildi.

\section{Bulgular}

Çalışmaya dahil edilen 100 hastanın 52'si erkek 48'i kadındı. Erkek hastaların yaşları 2495 (ort \pm SD: $56,6 \pm 15$ ), kadın hastaların yaşları 25-85 (ort \pm SD: 52,7 \pm 16 ) arasında değişmekte idi. Çalışmaya alınan 100 hastanın 24'ü (\%24) 20-44 yaş, 46'sı (\%46) 45-64 yaş ve 30'u (\%30) 65 yaş ve üzerindeydi. PİOPED kriterlerine göre emboli riski ile yaş arasındaki ilişkiye bakıldığında; PE olmayan grupta yaş 54,3 \pm 16 ; düşük olasılıklı grupta yaş 55,5 \pm 13 ; yüksek olasılıklı grupta yaş $54,0 \pm 17$ ve PE var olan grupta 56,2 \pm 14 olarak bulundu. Hastalardan 20-44 yaş grubunda olanlarda D-dimer düzeyleri ortalamas1 $4,21 \pm 4 \mu \mathrm{g} / \mathrm{mL} ; 45-64$ yaş grubunda olanlarda $5,16 \pm 10 \mu \mathrm{g} / \mathrm{mL} ; 65$ yaş ve üzerinde olanlarda $4,8 \pm 4 \mu \mathrm{g} / \mathrm{mL}$ idi ( $\mathrm{p}>0,05)$.

Pulmoner emboli olmayan 65 hastada D-dimer: 4,39 $\pm 9 \mu \mathrm{g} / \mathrm{mL}$; düşük olasıllkl1 20 hastada D-dimer: 4,57 $\pm 4 \mu \mathrm{g} / \mathrm{mL}$; orta olas1lıkl1 6 hastada D-dimer: $6,39 \pm 6 \mu \mathrm{g} / \mathrm{mL}$; yüksek olasılıklı PE olan 9 hastada D-dimer: $8,0 \pm 8 \mu \mathrm{g} / \mathrm{mL}$ olarak bulundu (Tablo 1). Orta ve yüksek olasılıklı PE olan hastalarda ortalama D-dimer düzeyleri, PE olmayan ve düşük olasılıklı gruba göre daha yüksek bulunmuş olmasına rağmen istatistiksel olarak anlamlı değildi. Perfüzyon defekti izlenen segment sayısına göre; perfüzyon defekti olmayan 22 hastada D-dimer: 3,01 $\pm 4 \mu \mathrm{g} / \mathrm{mL}$; subsegmenter defekti olan 8 hastada Ddimer: 3,62 $\pm 3 \mu \mathrm{g} / \mathrm{mL} ; 1$ segment defekti olan 14 hastada D-dimer: $10,7 \pm 18 \mu \mathrm{g} / \mathrm{mL} ; 2$ segment defekti olan 11 hastada D-dimer: 3,16 $\pm 2 \mu \mathrm{g} / \mathrm{mL} ; 3$ segment defekti olan 10 hastada D-dimer: 3,86 $\pm 3 \mu \mathrm{g} / \mathrm{mL}$; 3 'ten fazla segment defekti olan 35 hastada D-dimer: $4,66 \pm 5 \mu \mathrm{g} / \mathrm{mL}$ olarak bulundu (Tablo 2$)(\mathrm{p}<0,05)$. 
Cinsiyete ve PE varlığı/yokluğuna göre D-dimer düzeylerine bakıldığında; PE olmayan 36 kadın hastanın ortalama D-dimer düzeyi 3,37 $\pm 3 \mu \mathrm{g} / \mathrm{mL}$ iken, PE olan 3 hastanın ortalama D-dimer düzeyi 3,37 $\pm 3 \mu \mathrm{g} / \mathrm{mL}$ idi $(\mathrm{p}>0,05)$. Erkek hastalarda ise; PE olmayan 29 ortalama D-dimer düzeyi 5,67 $\pm 13 \mu \mathrm{g} / \mathrm{mL}$ iken, PE olan 6 hastanın ortalama D-dimer düzeyi $6,92 \pm 6 \mu \mathrm{g} / \mathrm{mL}$ idi $(\mathrm{p}>0,05)$.

Tablo 1. D-dimer düzeyleri ile pulmoner emboli olasılığı arasındaki ilişki (p>0,05).

\begin{tabular}{lllll}
\hline & PE yok & Düşük olasılık & Orta olasılık & Yüksek olasılık \\
\hline Hasta say1s1 & 65 & 20 & 6 & 9 \\
D-dimer $(\mu \mathrm{g} / \mathrm{mL})$ & $4,39 \pm 9$ & $4,57 \pm 4$ & $6,39 \pm 6$ & $8,0 \pm 8$ \\
\hline
\end{tabular}

Tablo 2. D-dimer düzeyleri ile perfüzyon sintigrafisindeki perfüzyon defekti sayıları arasındaki ilişki $(\mathbf{p}<\mathbf{0 . 0 5})$.

\begin{tabular}{lllllll}
\hline & $\begin{array}{l}\text { Perfüzyon } \\
\text { defekti yok }\end{array}$ & Subsegmenter & $\begin{array}{l}\text { 1 segment } \\
\text { tutulum }\end{array}$ & $\begin{array}{l}\text { 2 segment } \\
\text { tutulum }\end{array}$ & $\begin{array}{l}\text { 3 segment } \\
\text { tutulum }\end{array}$ & $\begin{array}{l}\text { 3'ten fazla } \\
\text { segment tutulum }\end{array}$ \\
\hline Hasta say1s1 & 22 & 8 & 14 & 11 & 10 & 35 \\
D-dimer $(\mu \mathrm{g} / \mathrm{mL})$ & $3,01 \pm 4$ & $3,62 \pm 3$ & $10,7 \pm 18,4$ & $3,16 \pm 2$ & $3,86 \pm 3$ & $4,66 \pm 5$ \\
\hline
\end{tabular}

\section{Tartışma}

Pulmoner emboli (PE) ölüm riskinin yüksek olması ve PE kuşkusu ile başlanan antikoagülan tedavinin yüksek kanama riski taşıması nedeniyle, tanının en kısa sürede doğrulanmasını ya da dışlanmasını gerektiren bir kardiovasküler hastalıktır. Pulmoner emboli derin ven trombozunun (DVT) bir komplikasyonudur. Kan dolaşımındaki staz, vasküler duvarlarda hasar ve hiperkoagülabilite DVT'nin patogenezinde yatan üç temel sebeptir. Teşhiste klinik şüphe önemlidir. Bizim çalışmamızda PE ile yaş grupları arasında istatistiksel olarak anlamlı farklılık olmamakla birlikte, yapılan çalışmalarda PE insidansının yaşla birlikte arttığı bildirilmektedir [6]. Bununla birlikte D-dimer seviyeleri de yaşla birlikte artış gösterir. $\mathrm{Bu}$ nedenle hastanın ileri yaşlarda olması $\mathrm{D}$-dimer düzeylerinin sensitivitesini azaltır [7]. Pulmoner anjiografi PE tanısı için altın standart olmakla birlikte, invaziv bir uygulama yöntemi olduğu için rutinde tercih edilmez. $\mathrm{Bu}$ nedenle, fizik muayene, risk faktörlerinin değerlendirilmesi, EKG, direk grafi ve laboratuvar testlerinin ardından, dopler ultrasonografi ve bilgisayarlı tomografik pulmoner anjiografi klinik olarak şüphelenilen hastalarda ilk yapılacak tetkiklerdendir. Son yıllarda uygulama alanına giren çok kesitli bilgisayarlı tomografiler sayesinde $1 \mathrm{~mm}$ veya daha ince kesitler alınarak yapılan incelemeler ile çok küçük çaplı arterlerdeki emboliler bile tespit edilebilmektedir. Bu nedenle çoğu merkezde PE şüpheli hastalarda kontrastlı BT pulmoner anjiografi ilk tercih edilen yöntemdir. Ancak unutulmaması gereken bir konu şudur ki; pulmoner embolilerin \%60-80 gibi büyük bir çoğunluğu küçüktür ve klinik belirti vermez. Zamanla organize olarak damar duvarına kaynaşırlar. $\mathrm{Bu}$ durum, BT ile saptanmış olan bu çok küçük embolilerin aslında rezorbe olup kaybolacağı anlamına gelebilir. Belki gerçekte önemli olan emboliyi tespit etmek değil, akciğerde meydana getirdiği etkiyi tespit etmektir. Çünkü akciğerler sadece pulmoner arterlerle değil aynı zamanda bronşial arterler tarafından ve alveollerden direk hava ile beslenmektedir. Eğer bronşial sirkülasyon normalse ve yeterli ventilasyon sağlanıyorsa kan akımında azalma doku nekrozuna neden olmaz [8]. Sintigrafik görüntüleme yöntemleri asıl olarak, anatomiyi değil fizyolojiyi görüntüler ve bu nedenle önemlidir. Pulmoner dolaşımda var olan bir embolinin akciğerde ilgili segmentte defekt oluşturup oluşturmaması ve klinik bulgular, tedavi kararının verilmesinde önemli olabilmektedir. Sonuçta bilgisayarlı tomografide tespit edilen her emboliyi tedavi etmek gerekir mi sorusu da akla gelmektedir. $\mathrm{Bu}$ nedenle PE şüphesi olan hastada anti koagülan ve antiagregan tedavinin morbidite ve mortalitesi de dikkate alınarak tanı doğruluğu açısından daha hassas yaklaşılmalıdır. Bizim çalışmamızda, kliniğimize PE şüphesi ile gelen hastalardan 65'inde (\%65) D-dimer seviyesi normalden yüksek olmakla birlikte $(4,39 \pm 9 \mu \mathrm{g} / \mathrm{mL})$ perfüzyon/ventilasyon sintigrafisinde perfüzyon defekti yoktu. 
Belki de bu hastalardaki D-dimer yüksekliği gerçekten küçük de olsa bir emboli varlığına bağlıydı. Ancak ne yazık ki, çalışmamızın retrospektif olması dışındaki en büyük kısıtlılı̆̆ bu hastaların BT görüntülerine ulaşılamamış olmasıdır.

Bilgisayarlı tomografi görüntülemede iyot içerikli kontrast kullanımına bağlı alerjik reaksiyonlar, kontrastla tetiklenen nefropati ve radyasyon dozunun fazlalığı nedeniyle ventilasyon/perfüzyon sintigrafisi, bilgisayarlı tomografiye alternatif olarak kullanılabilecek görüntüleme yöntemidir. Perfüzyon sintigrafisinde amfizem, bronşektazi, akciğer absesi, bronş obstrüksiyonuna neden olan akciğer kanseri, plörezi, pnömotoraks, yüksek diyafragma ve pulmoner damar problemleri perfüzyon defektine neden olabilir. Akut pulmoner embolide ventilasyon bozulmayacağ normaldir (mismatched defekt-uyumsuz defekt). Ventilasyon sintigrafisi, akut PE dışındaki durumlarda, perfüzyon defektlerine neden olabilen, ancak ventile olmayan anormal akciğer sahalarını göstererek perfüzyon sintigrafisinin duyarlılığını artırır. Herhangi bir perfüzyon defekti izlenmeyen normal perfüzyon sintigrafisi, PE'yi ekarte ettirir. Tc99m-LyoMAA ile perfüzyon sintigrafisinde en az bir perfüzyon defekti ile birlikte normal ventilasyon sintigrafisi $\% 85-90$ oranında yüksek olasilıkla pulmoner emboliyi gösterir [9].

Bununla birlikte V/P sintigrafisinin de bazı kısıtlılıkları vardır. Bunlardan biri V/P sintigrafisinin iki gün protokolü ile uygulanabilirliğidir. Diğeri ise, anatomik olarak segmentlerin detaylı olarak tespit edilememesi ve bazı segmentlerde de shine-through nedeniyle embolik defektin tam olarak ayırımının yapılamamasıdır. Örnek olarak sağ akciğer alt lob medial bazal segment planar çekimlerde genellikle görüntülenemez. Bu sorun V/P sintigrafisinin sensitivitesi ve spesifitesi yüksek olan SPECT/BT ile elde edilmesi sayesinde aşılabilmektedir [10]. Ventilasyon/perfüzyon sintigrafisinin yorumlanmasında PİOPED kriterleri kullanılmaktadır. Bununla birlikte literatürde PE teşhisinde kullanılan kriterler arasında da çeşitlilik vardır. Le Roux ve ark. [11] 1 segmental defekt veya 2 subsegmental uyumsuz (mismatch) defektin akut pulmoner emboliyi ayırt edebileceğini rapor etmişlerdir. Oysa diğer bir makalede boyutu ne olursa olsun 1 uyumsuz segmental defektin PE için şüpheli olduğu rapor edilmiştir [12]. Ventilasyon/perfüzyon sintigrafisi akut/kronik emboli ayırımını yapamamaktadır. Çalışmamızda ilginç olarak perfüzyon sintigrafisine göre; D-dimer seviyesi 1 segmentte perfüzyon defekti olan grupta yüksek, buna karşın 3'ten fazla segmentte tutulum olan grupta ise daha düşük olarak bulundu. Bu durum, tek segment tutulumu olan vakaların gerçek akut PE ile uyumlu vakalar olması ve daha fazla sayıda segment tutulumu olanların ise daha çok kronik hastalıkları bulunan vakalar olduğunu düşündürmektedir. Tam tanımlanamayan (indetermine) sonuçlar klinisyenler açısından planar V/P sintigrafisinin popülaritesini azaltmaktadır [13]. Her ne kadar günümüzde planar V/P sintigrafisi genellikle kontrast maddeye alerjisi olan, ciddi böbrek yetmezliği bulunan ve ekipman eksikliği nedeniyle BT anjiografi yapılamayan hastalarda alternatif olarak kullanılmakta ise de, tanısal doğruluğu yüksek olan SPECT/BT sistemlerinin gelişimi sayesinde V/P sintigrafisinin duyarlılı̆̆ 1 artırılmıştır [14].

Yaklaşık 20 yıl önce derin ven trombozu tanısı olan olgularda alternatif veya destekleyici test olarak sunulmuş olan D-dimer, yüksek negatif öngörüsü ve endojen fibrinolitik aktiviteyi göstermesi nedeniyle pulmoner emboliyi ekarte etmede rutinde halen kullanılan basit ve non-invaziv yöntemdir [15]. Literatürde D-dimer düzeyleri ile PE arasındaki ilişkiyi araştıran çok sayıda araştırma bulunmaktadır. Aujesky ve ark. [16] PE'de yüksek D-dimer seviyelerinin artmış mortalite riski ile ilişkili olduğunu rapor etmişlerdir. Ayrıca Galle ve ark. [17] yaptıkları çalışmalarında, plazma D-dimer düzeyi ile PE büyüklügüunün ilişkili olduğunu rapor etmişlerdir. Eichinger ve ark. [18] yaptıkları prospektif çalışmalarında, D-dimer düzeyleri ile venöz tromboembolinin rekürrens riski arasındaki ilişkiyi araştırmışlar ve D-dimer düzeyi $250 \mathrm{ng} / \mathrm{mL}$ 'den düşük olanlarda rekürrens riskinin daha az olduğunu göstermişlerdir. Bununla birlikte, maligniteler, tüketim koagülopatisi (DIC), enfeksiyonlar, travma, cerrahi operasyonlar gibi fibrin yıkımının 
olduğu birçok durumda da artış gösterebilir [9]. Hatta son zamanlardaki yayınlarda kronik obstrüktif akciğer hastalıklarında da yüksek D-dimer seviyeleri rapor edilmektedir [19]. $\mathrm{Bu}$ nedenle sensitivitesi yüksek, ancak spesifitesi düşük bir testtir. D-dimer testinin negatif olması tromboz olasılığını ortadan kaldırmaktadır [20]. Bununla birlikte, yüksek klinik risk taşıyan olgularda negatif D-dimer düzeyleri dikkate alınmamalıdır [3]. Ancak testin pozitif olması DVT tanısı ile birlikte diğer tanı olasılıklarını da gündeme getirmektedir. Ayrıca ileri yaşlarda ve renal yetmezlik durumunda D-dimer seviyesinde artış olduğu da bilinmektedir [7,21]. Bizim yaptığımız çalışmada ise, orta ve yüksek olasılıklı PE olan hastalarda ortalama D-dimer düzeyleri, PE olmayan ve düşük olasılıklı gruba göre daha yüksek bulunmuş olmasına rağmen istatistiksel olarak anlamlı değildi.

D-dimer düzeyi PE tanısının desteklenmesinde önemli bir antitedir. D-dimer düzeyi ile ventilasyon/perfüzyon sintigrafisindeki sintigrafik bulguların ilişkisini incelediğimiz çalışmamızın sonucunda, D-dimer seviyesi bir segmentte perfüzyon defekti olan grupta yüksek, buna karşın üçten fazla segmentte tutulum olan grupta ise daha düşük olarak bulundu. $\mathrm{Bu}$ durum, tek segment tutulumu olan vakaların gerçek akut PE ile uyumlu vakalar olması ve daha fazla sayıda segment tutulumu olanların ise daha çok kronik hastalıkları bulunan vakalar olduğunu düşündürmektedir.

\section{Kaynaklar}

1. Ödev K. Toraks Radyolojisi. Bölüm 19. Pulmoner Vasküler Hastalıklar. Nobel Tip Kitabevleri Ltd. Şti. İstanbul 2005; 331-55.

2. Arseven O, Sevinç C, Alataş F, Ekim N, Erkan L, Fındık S. Türk Toraks Derneği pulmoner tromboembolizm tanı ve tedavi uzlaşı raporu. Turk Toraks Derg 2009; 10: $1-47$.

3. Uresandi F, Blanquer J, Conget F, de Gregorio MA, Lobo JL, Otero R, Pérez Rodríguez E, Monreal M, Morales P. Guidelines for the diagnosis, treatment, and follow-up of pulmonary embolism. Arch Bronconeumol 2004; 40: 580-94.

4. Bělohlávek J, Dytrych V, Linhart A. Pulmonary embolism, part I: Epidemiology, risk factors and risk stratification, pathophysiology, clinical presentation, diagnosis and nonthrombotic pulmonary embolism. Exp Clin Cardiol 2013; 18: 129-38.

5. Gottschalk A, Sostman HD, Coleman RE, Juni JE, Thrall J, McKusick KA, Froelich JW, Alavi A. Ventilation-perfusion scintigraphy in the PIOPED study. Part II. Evaluation of the scintigraphic criteria and interpretations. J Nucl Med 1993; 34: 1119-26.

6. Duru S, Ergün R, Dilli A, Kaplan T, Kaplan B, Ardıç S. Pulmoner embolide klinik, laboratuvar ve bilgisayarlı tomografi pulmoner anjiyografi sonuçları: 205 hastanın retrospektif değerlendirmesi. Anadolu Kardiyol Derg 2012; 12: 142-9.

7. Douma RA, Tan M, Schutgens RE, Bates SM, Perrier A, Legnani C, Biesma DH, Ginsberg JS, Bounameaux H, Palareti G, Carrier M, Mol GC, Le Gal G, Kamphuisen PW, Righini M. Using an age-dependent D-dimer cut-off value increases the number of older patients in whom deep vein thrombosis can be safely excluded. Haematologica 2012; 97: 1507-13.

8. Kumar V, Cotran RS, Robbins SL. Temel Patoloji (Basic Pathology). Çevikbaş U (Çeviri Editörü). Akciğerler ve Üst Solunum Yolları. 13. Bölüm. Nobel Tıp Kitabevleri Ltd. Şti 2000; 393-438.

9. Huisman MV, Klok FA. How I diagnose acute pulmonary embolism. Blood. 2013; 121: 4443-8.

10. Roach PJ, Schembri GP, Bailey DL. V/Q scanning using SPECT and SPECT/CT. J Nucl Med 2013; 54: 1588-96.

11. Le Roux PY, Robin P, Delluc A, Abgral R, Le Duc-Pennec A, Nowak E, Couturaud F, Le Gal G, Salaun PY. V/Q SPECT interpretation for pulmonary embolism diagnosis: Which criteria to use? J Nucl Med 2013; 54: 1077-81.

12. Howarth DM, Booker JA, Voutnis DD. Diagnosis of pulmonary embolus using 
ventilation/perfusion lung scintigraphy: more than 0.5 segment of ventilation/perfusion mismatch is sufficient. Intern Med J 2006; 36: 281-8.

13. Dursun AB, Güven SF, Saka D, Sarıŏlu N, Şipit T. Klinik Pratikte Pulmoner Tromboemboliye Yaklaşım (Approach to the Pulmonary Thromboembolism in Clinical Practice).Tuberk ve Toraks 2001; 49: 464-70.

14. Mahdavi R, Caronia J, Fayyaz J, Panagopoulos G, Lessnau KD, Scharf SC, Mina B, Allred C, DiFabrizio L. Agreement between SPECT V/Q scan and CT angiography in patients with high clinical suspicion of PE. Ann Nucl Med 2013; 27: 834-8.

15. Gutte H, Mortensen J, Jensen CV, von der Recke P, Petersen CL, Kristoffersen US, Kjaer A. ANP, BNP and D-dimer predict right ventricular dysfunction in patients with acute pulmonary embolism. Clin Physiol Funct Imaging 2010; 30: 466-72.

16. Aujesky D, Roy PM, Guy M, Cornuz J, Sanchez O, Perrier A. Prognostic value of D-dimer in patients with pulmonary embolism. Thromb Haemost 2006; 96 : 478-82.

17. Galle C, Papazyan JP, Miron MJ, Slosman D, Bounameaux H, Perrier A. Prediction of pulmonary embolism extent by clinical findings, D-dimer level and deep vein thrombosis shown by ultrasound. Thromb Haemost 2001; 86: 1156-60.

18. Eichinger S, Minar E, Bialonczyk C, Hirschl M, Quehenberger P, Schneider B, Weltermann A, Wagner O, Kyrle PA. D-dimer levels and risk of recurrent venous thromboembolism. JAMA 2003; 290: 1071-4.

19. Akpinar EE, Hoşgün D, Doğanay B, Ataç GK, Gülhan M. Should the cut-off value of D-dimer be elevated to exclude pulmonary embolism in acute exacerbation of COPD? J Thorac Dis 2013; 5: 430-4.

20. Noyan T. Klinik Tanı ve Laboratuvar Pratiğinde D-dimer Testi (D-dimer Testing in Clinical Diagnosis and Laboratory Practice). Turk Klinik Biyokimya Derg 2012; 10: 35-40.

21. Lindner G, Funk GC, Pfortmueller CA, Leichtle AB, Fiedler GM, Schwarz C, Exadaktylos AK, Puig S. D-Dimer to rule out pulmonary embolism in renal insufficiency. Am J Med. 2013; 16: 1071-1. 